\title{
Tetrahydro-3-pyranon: Regioselektivität bei der Bildung reaktiver Intermediate
}

\author{
Klaus Th. Wanner \\ Institut für Pharmazie und Lebensmittelchemie der Universität München, \\ Sophienstr. 10, 8000 München 2.
}

Eingegangen am 9. Juni 1987

Die kinetisch kontrollierte Deprotonierung des Pyranons 1 sowie des Pyranimins 3 erfolgt bevorzugt in 4-Position des Pyranringes. Diese Orientierung wird in noch stärkerem Maße unter thermodynamischer Kontrolle beobachtet. Die Regioselektivitäten werden mit denen früher untersuchter Pyranenamine 8-11 und -silylenolether 7 verglichen, Faustregeln für die Bildung der Regioisomere vorgeschlagen.
Tetrahydropyran-3-one: Regioselectivity in the Formation of Reactive Intermediates

Kinetically controlled deprotonations of tetrahydropyranone 1 and tetrahydropyranimine 3 occur preferentially at position 4 of the pyran ring. The same orientation is even more preferred under thermodynamic control. The regioselectivities are compared with those of the earlier investigated dihydropyranamines 8-11 and of the silyl enole ether 7. A rule of thumb for the ratio of regioisomers is proposed.
Der Einsatz des Tetrahydro-3-pyranons (1) bietet einen bequemen und variablen Zugang $\mathrm{zu}$ verschiedenartigen pyrananellierten Heterocyclen. Wir haben gezeigt ${ }^{1}$, daß durch richtige Wahl reaktiver Intermediate Substituenten selektiv in 2- und 4-Stellung des Pyranringes dirigiert werden können. Es gelingt so, regioisomere Heterocyclen gezielt zu synthetisieren. Wir haben jetzt auch das Imin 3 bzw. seine metallierten Derivate $4 \Delta^{2}$ bzw. $4 \Delta^{3}$ und die Lithiumenolate $6 \Delta^{2}$ und $6 \Delta^{3}$ in unsere Untersuchungen einbezogen.

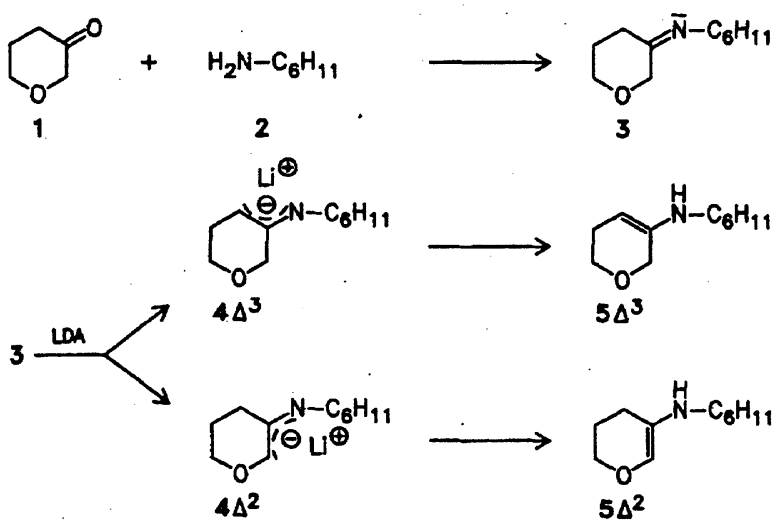

Bei der Reaktion von 1 mit Cyclohexylamin (2) unter $\mathrm{Zu}$ satz von Molekularsieb entstand das Imin 3 in 73proz. Ausbeute. Die kinetisch kontrollierte Deprotonierung gelang mit Lithiumdiisopropylamid bei $-78^{\circ} \mathrm{C}$. Zur Analyse der Isomerenzusammensetzung haben wir nach $K$ norr ${ }^{2)}$ die Lithioenamine mit $\mathrm{CH}_{3} \mathrm{OH}$ regiospezifisch $\mathrm{N}$-protoniert und die ${ }^{1} \mathrm{H}$ NMR-Spektren der so erzeugten Enamine gemessen. Auffallend sind Signale bei $\delta=4.23(\mathrm{t})$ und $\delta=5.78 \mathrm{ppm}(\mathrm{s})$, die wir den Olefinprotonen der Strukturisomere $5 \Delta^{3}$ bzw. $5 \Delta^{2}$ zuordnen. Die Integration der Signale ergab ein 37/63-Verhältnis für die Enamine $5 \Delta^{2} / 5 \Delta^{3}$ und damit auch für die Lithioenamine $4 \Delta^{2} / 4 \Delta^{3}$. Außerdem waren noch zwei Signale bei $\delta$ $=3.75$ (s) und $\delta=3.78 \mathrm{ppm}$ (s) zu beobachten, die von den Protonen in 2- und 4-Stellung des Ausgangsmaterials 3 herrühren, in das die instabilen Enamine 5 wieder umlagern.
Zur Einstellung eines thermodynamisch kontrollierten Gleichgewichts haben wir. 3 in geringem Uberschuß mit LDA deprotoniert und das Gemisch vor der Protonierung mit $\mathrm{CH}_{3} \mathrm{OH} 2 \mathrm{~h}$ bei Raumtemperatur gerührt; überschüssiges 3 sollte als schwache Säure die Gleichgewichtseinstellung katalysieren. Im ' ${ }^{1} \mathrm{H}-\mathrm{NMR}$-Spektrum war dann nahezu ausschließlich das Signal für das $\Delta^{3}$-Isomere bei $\delta=4.23 \mathrm{ppm}(\mathrm{t})$ nachzuweisen $\left(5 \Delta^{3} / 5 \Delta^{2}>95 / 5\right)$. Folglich ist die thermodynamisch stabilere Form der Lithioenamine, diejenige $\left(4 \Delta^{3}\right)$ mit der Doppelbindung, respektive negativen Ladung, abgewandt vom Heteroatom. Bedenkt man, daß metallierte Enamine (i. Vgl. zu Enaminen) glatt auch mit weniger reaktiven Alkylhalogeniden ${ }^{3)}$ und selbst mit Carbonsäureamiden") reagieren, solite das eine brauchbare Methode sein, um entsprechende Gruppen selektiv in 4-Stellung einzuführen.

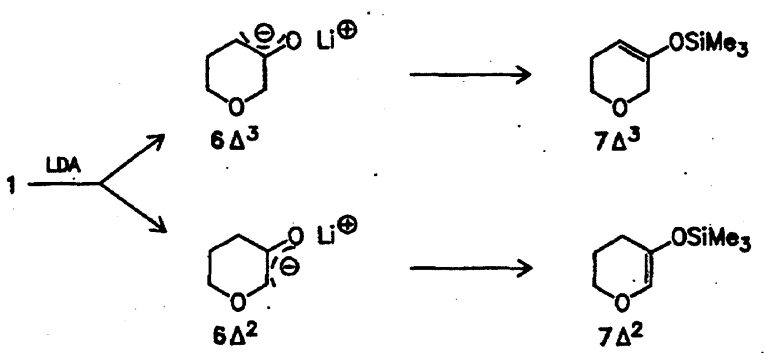

Nach der kinetisch kontrollierten Deprotonierung des 3Pyranons 1 bei $-78^{\circ} \mathrm{C}$ und dem Abfangen mit Trimethylchlorsilan in Anlehnung an House ${ }^{5)}$, entstand ein Gemisch von $7 \Delta^{2} / 7 \Delta^{3}=23 / 77$. Zur Herstellung von Gleichgewichtsbedingungen haben wir 1 bei $-78^{\circ} \mathrm{C}$ mit etwas weniger als der äquimolaren Menge LDA deprotoniert, und zur Äquilibrierung ${ }^{\text {( }}$ bei verschiedenen Temp. und verschieden lang gerührt $\left(-78^{\circ} \mathrm{C}, 2 \mathrm{~h} ;-78^{\circ} \mathrm{C}, 5 \mathrm{~h} ;-78^{\circ} \mathrm{C}, 2 \mathrm{~h}\right.$ und $-45^{\circ} \mathrm{C}$, $0.75 \mathrm{~h} ;-20^{\circ} \mathrm{C}, 1 \mathrm{~h}$ ). Dann wurde bei $-78^{\circ} \mathrm{C}$ silyliert. Mit zunehmender Äquilibrierungsdauer und -temp. erhöhte sich $\operatorname{der}$ Anteil des $\Delta^{3}$-isomeren Silylenolethers $(82,85,93,>$ $95 \%), 7 \Delta^{2}$ war schließlich ${ }^{1} \mathrm{H}$-NMR-spektroskopisch nicht mehr nachweisbar. Also ist auch das Isomer von 6 mit der 
Doppelbindung in $\Delta^{3}$-Position thermodynamisch begünstigt, vorausgesetzt, die beobachteten Veränderungen beruhen nicht auf Folgereaktionen?"

Wie die folgende U'bersicht zeigt, stehen nun reaktive $\Delta^{2}$ und $\Delta^{3}$-Intermediate von 1 zur Verfügung, die eine regioselektive Substitution der 2-bzw. 4-Position des 3-Pyranons 1 erlauben. protonierung oder durch eine rasche thermodynamische Åquilibrierung auf der Stufe eines intermediären Pyranenolats (oder -enols) vor der Silylierung erklärbar, eventuell in Kombination mit unterschiedlichen Silylierungsgeschwindigkeiten. Beides ist mit den oben beschriebenen Ergebnissen der Lithiumenolatbildung $(1 \rightarrow 6)$ vereinbar; die gezeigt ha-

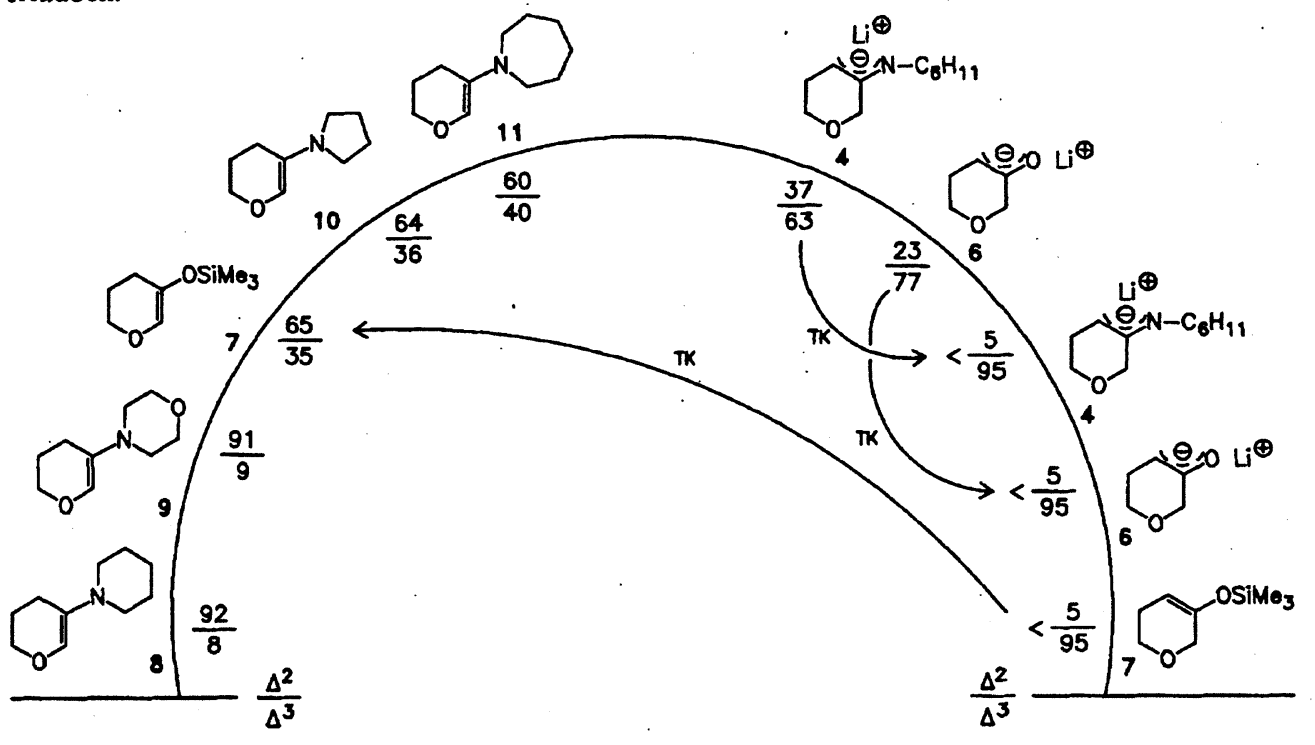

TK = Thermodynamische Kontrolle

Die Piperidin- und Morpholinenamine 8 bzw. 9 liegen fast ausschließlich als $\Delta^{2}$-Isomer vor; sie entstehen thermodynamisch kontrolliert, wie aufgrund der Synthesebedingungen ${ }^{1)}$ anzunehmen ist ${ }^{8)}$.

Den Silylenolether mit der Doppelbindung in 3-Stellung $7 \Delta^{3}$ haben wir isomerenrein $\left(\mathbf{7} \Delta^{\mathbf{2}}{ }^{1} \mathrm{H}-\mathrm{NMR}\right.$-spektroskopisch nicht mehr nachweisbar) und in $79 \%$ Ausbeute aus 1 mit $\mathrm{ClSiMe}_{3} / \mathrm{NEt}_{3}$, in THF $(\Delta \mathrm{T})$ dargestellt ${ }^{1)}$. Unter den von House $^{3)}$ angegebenen Bedingungen (ClSiMe ${ }_{3}, \mathrm{NEt}_{3}, \mathrm{DMF}$, $\Delta T$ ) bilden sich bei Ketonen, die in $\alpha$-Stellung nicht mit einem Heteroatom substituiert sind, im thermodynamischen Gleichgewicht befindliche Mischungen der Silylenolether, wobei die Gleichgewichtslage dieser säurekatalysierten Äquilibrierung qualitativ der der Enolate" entspricht.

Beim 3-Pyranon 1 haben dagegen unsere Silylierungsversuche (DMF, ClSiMe ${ }_{3} / \mathrm{NEt}_{3}$ ) gezeigh, daß zwar erst der $\Delta^{3}$ Silylenolether $7 \Delta^{3}$ entsteht, sich bei längerem Erhitzen jedoch das $\Delta^{2}$-Isomere bildet, bis das Verhältnis 65/35 (7 $\Delta^{2} /$ $7 \Delta^{3}$ ) beträgt. Auch der reine, isolierte Silylenolether $7 \Delta^{3}$, in DMF in Gegenwart äquimolarer Mengen $\mathrm{NEt}_{3} \cdot \mathrm{HCl}$ zum Rückfluß erhitzt ${ }^{1)}$, isomerisiert zum $\Delta^{2}$-Isomer $7 \Delta^{2}$ (nach $3 \mathrm{~h}$ $7 \Delta^{2} / 7 \Delta^{3} \sim 64: 36$ ) (Die Isomerisierung war geringfügig von einer Spaltung zum 3-Pyranon 1 begleitet). Die selektive Silylierung des 3-Pyranons zum $\Delta^{3}$-Silylenolether $7 \Delta^{3}$ (mit $\mathrm{NEt}_{3} / \mathrm{ClSiMe}_{3}$ in THF) kann deswegen nicht die Folge einer thermodynamischen Kontrolle sein, und das für ein thermodynamisches Gleichgewicht von Hirsch berichtete ${ }^{\text {9) }}$ Verhältnis von 11:89 $\left(7 \Delta^{2} / 7 \Delta^{3}\right)$ erscheint fragwürdig. Die ausschließliche Bildung von $7 \Delta^{3}$ ist durch eine regioselektive De- ben, da $\mathrm{B}$ die Bildung des $\Delta^{3}$-Isomeren unter kinetischen und - noch deutlicher - unter thermodynamischen Bedingungen überwiegt.

Das Imin 3 ist mit seiner kinetischen und thermodynamischen Acidität von 63/37 bzw. > 95/5 für $4 \Delta^{3} / 4 \Delta^{2}$ dem Pyranon 1 ähnlich. In der Formelübersicht sind auch die Pyrrolidin- und Perhydroazepinenamine 10 und 11 aufgeführt. Wegen der ungünstigen Isomerenverhältnisse sind sie präparativ wenig brauchbar, sie lassen aber erkennen, daß mit zunehmender Elektronendichte am B-C-Atom die Doppelbindung einer Konjugation mit dem Ringsauerstoff ausweicht, denn in der Reihe Morpholin-, Piperidin- Perhydroazepinund Pyrrolidinenamin steigt die Elektronendichte am B-CAtom (und somit die Reaktivität der Enamine gegenüber elektrophilen Reagenzien ${ }^{10}$ ) an. Besonders ausgeprägt ist diese Tendenz "negative Ladung $\rightarrow \Delta^{3}$-Doppelbindung " bei den im thermodynamischen Gleichgewicht befindlichen $\mathrm{Li}$ thiumenolaten und enaminen 6 bzw. 4 , bei denen das $\Delta^{3}$. Isomer eindeutig dominiert. Wird die Elektronendichte durch Silylierung zu 7 dagegen verringert, bewegt sich (unter Äquilibrierungsbedingungen) die Doppelbindung wieder zum Sauerstoff hin.

Bei der thermodynamisch kontrollierten Bildung von $\Delta^{2}$ oder $\Delta^{3}$-Dihydropyranderivaten sind folgende Faustregeln nützlich:

Bildung eines Derivats mit

„Carbanionencharalkter" begünstigt das $\Delta^{3}$-Isomer (z. B. 6) (höhere Elektronendichte) 
Bildung eines Derivats mit "Alkencharakter' begünstigt das $\Delta^{2}$-Isomer (z. B. 8) (niedrigere Elektronendichte)

Als Ausnahme von dieser Vorstellung ist ein Enolacetat des 3-Pyranons (1) anzusehen, denn unter thermodynamischen Bedingungen soll ausschließlich das $\Delta^{3}$-Isomer entstehen ${ }^{11)}$.

Herrn Prof. F. Eiden danke ich für die großzügige Förderung, Frau Kärtner fuir die engangierte Mitarbeit beim Experimentieren.

\section{Experimenteller Teil}

IR-Spektren: Acculab 6(Beckman). - 'H-NMR-Spektren: T 60 und A 60 (Varian); 8-Skala (ppm), TMS int. Stand. - MS: CH7 (Varian). Sämtliche Umsetzungen wurden unter $\mathrm{N}_{2}$ durchgeführt.

N-Cyclohexyl-5,6-dihydro-2H-pyran-3(4H)-imin (3)

Eine Mischung aus $10.0 \mathrm{~g}(100 \mathrm{mmol}) 1,10.91 \mathrm{~g}(110 \mathrm{mmol}) 2$ und $50 \mathrm{ml}$ absol. Benzol wurde nach Zusatz von $20 \mathrm{~g}$ Molekularsieb (4A) $60 \mathrm{~h}$ bei Raumtemp. gerührt. Es wurde filtriert und anschließend fraktioniert destilliert. Farblose Flüssigkeit; Sdp.0.05 66-69.5 ${ }^{\circ} \mathrm{C}$, Ausb. 13.26 g (73 \%). $\mathrm{C}_{21} \mathrm{H}_{19} \mathrm{NO}(181.3)$ Ber. C $72.9 \mathrm{H} 10.56 \mathrm{~N} 7.7 \mathrm{Gef}$. C 72.8 H $10.58 \mathrm{~N} 7.7$ Mol.-Masse 181 (ms). - IR (Film): 1665, $1445 \mathrm{~cm}^{-1}$. - 'H-NMR ( $\mathrm{CDCl}_{3}$ ): $0.85-2.10(\mathrm{~m}, 12 \mathrm{H}), 2.48$ und $2.50(2 \mathrm{t}, \mathrm{J}=7 \mathrm{~Hz}$ und $\mathrm{J}=6.5 \mathrm{~Hz}$, zusam$\left.\operatorname{men} 2 \mathrm{H}, \mathrm{O}-\mathrm{CH}_{2}-\mathrm{CH}_{2}-\mathrm{CH}_{2}\right), 3.20-3.65(\mathrm{~m}, 1 \mathrm{H},=\mathrm{NCH}), 3.75$ und 3.78 $\left(2 \mathrm{t}, \mathrm{J}=5.5 \mathrm{~Hz}\right.$ und $\mathrm{J}=5 \mathrm{~Hz}$, zusammen $\left.2 \mathrm{H}, \mathrm{OCH}_{2} \mathrm{CH}_{2}\right), 4.03(\mathrm{~s}, 0.7 \mathrm{H}$, $\left.\mathrm{OCH}_{2} \mathrm{C}=\mathrm{N}\right), 4.20\left(\mathrm{~s}, 0.3 \mathrm{H}, \mathrm{OCH}_{2} \mathrm{C}=\mathrm{N}\right)$.

5-Cyclahexylamino-3,4-dihydro-2H-pyran $\left(5 \Delta^{2}\right)$ und 5-Cyclohexylamino-3,6-dihydro-2H-pyran $\left(5 \Delta^{3}\right)$

a) $0.32 \mathrm{~g}$ (3.15 mmol) Diisopropylamin in $3 \mathrm{ml}$ absol. THF wurden unter Eiskühlung mit $3.15 \mathrm{mmol} n$-Butyllithium (1.6 M, Hexanlösung) versetzt. Es wurde $15 \mathrm{~min}$. bei Raumtemp. gerührt, auf $-78^{\circ} \mathrm{C}$ gekühlt, tropfenweise mit $0.54 \mathrm{~g}$ ( $3.0 \mathrm{mmol}) 3$ (in $0.5 \mathrm{ml} \mathrm{THF}$ ) versetzt und noch $2 \mathrm{~h}$ bei $-78^{\circ} \mathrm{C}$ gehalten. $15 \mathrm{mmol}$ absol. $\mathrm{CH}_{3} \mathrm{OH}$ und $3 \mathrm{~g} \mathrm{~K}_{2} \mathrm{CO}_{3}$ wurden zugesetzt, es wurde filtriert, i. Vak. (15 Torr) sofort ein Großteil des Lösungsmittels abgedampth, absol. $\mathrm{CCl}_{4}$ zugesetzt und die Probe 'H-NMR-spektroskopiert. Danach lagen die Enamine $5 \mathrm{zu} .80 \%$ vor, 3 zu $20 \%$. ${ }^{-1} \mathrm{H}-\mathrm{NMR}\left(\mathrm{CCl}_{4}\right): 4.23\left(\mathrm{t}, \mathrm{J}=4 \mathrm{~Hz}, \mathrm{O}-\mathrm{CH}_{2}-\mathrm{CH}_{2}-\mathrm{CH}=\right.$ $\mathrm{C}-\mathrm{N}), 5.78(\mathrm{~s}, \mathrm{O}-\mathrm{CH}=\mathrm{C}-\mathrm{N}) .5 \Delta^{2} / 5 \Delta^{3}=37 / 63$.

b) $3.0 \mathrm{mmol}$ LDA-Lösung (Herstellung siehe a)) wurden bei $-20^{\circ} \mathrm{C}$ mit $0.57 \mathrm{~g}(3.15 \mathrm{mmol}) 3 \mathrm{in} 0.5 \mathrm{ml}$ absol. THF versetzt. Nach $2 \mathrm{~h}$ bei Raumtemp. wurde auf $-78^{\circ} \mathrm{C}$ gekühlt und wie bei a) weiter vorgegangen. Der Anteil von 3 im Vergleich zu 5 war $<10 \%$. ' 'H-NMR $\left(\mathrm{CCl}_{4}\right): 3.53\left(\mathrm{t}, \mathrm{J}=5.5 \mathrm{~Hz}, \mathrm{O}-\mathrm{CH}_{2}-\mathrm{CH}_{2}-\mathrm{CH}=\mathrm{C}\right), 3.68(\mathrm{~d}, \mathrm{~J}=1.5 \mathrm{~Hz}$, $\left.-\mathrm{OCH}_{2} \mathrm{C}=\mathrm{C}\right), 4.23\left(\mathrm{t}, \mathrm{J}=4 \mathrm{~Hz}, \overrightarrow{\mathrm{O}}-\mathrm{CH}_{2}-\mathrm{CH}_{2}-\mathrm{CH}=\mathrm{C}\right) .5 \Delta^{2} / 5 \Delta^{3}<5 /$ 95.

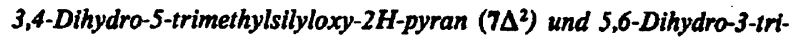
methylsilyloxy-2H-pyran $\left(7 \Delta^{3}\right)$

a) $0.56 \mathrm{~g}(5.5 \mathrm{mmol})$ Diisopropylamin in $5 \mathrm{ml}$ absol. THF wurden unter Eiskühlung mit $3.5 \mathrm{mmol}$ Methyllithium (1.6 M etherische Lösung) versetzt. Es wurde $15 \mathrm{~min}$ bei $0^{\circ} \mathrm{C}$ gerührt, anschließend auf $-78^{\circ} \mathrm{C}$ gekühlt, tropfenweise mit $0.5 \mathrm{~g}(5.0 \mathrm{mmol}) 1$ in $0.5 \mathrm{ml}$ absol. THF versetzt und $0.5 \mathrm{~h}$ bei $-78^{\circ} \mathrm{C}$ gerührt. Dann wurde eine vorbereitete $\mathrm{Mi}$ schung aus $0.90 \mathrm{~g}(8.33 \mathrm{mmol})$ Trimethylchlorsilan und $0.23 \mathrm{~g}$ (2.33 mmol) Triethylamin zugespritzt (unlösliches $\mathrm{NEt}_{3} \cdot \mathrm{HCl}$ war vorher durch Zentrifugieren abgetrennt worden). Nach 5 min wurde eine $2 \mathrm{ml}$ Probe am Rotationsverdampfer eingeengt, der Rückstand in $\mathrm{CDCl}_{3}$ aufgenommen und die Isomerenrelation ${ }^{2} \mathrm{H}$-NMR-spektroskopisch anhand der Intensität der Olefinsignale bei $\delta=6.27 \mathrm{ppm}(\mathrm{t}, \mathrm{J}=1.5 \mathrm{~Hz}$, $\left.7 \Delta^{2}\right)$ und $\delta=4.83-5.07\left(\mathrm{~m}, 7 \Delta^{3}\right)$ bestimmt. $7 \Delta^{2} / 7 \Delta^{3}=23 / 77$.

b). Analog zu a) wurden $5.0 \mathrm{mmol}$ LDA mit $0.55 \mathrm{~g}(5.5 \mathrm{mmol}) 1$ umgesetzt. Vor der Silylierung wurde $2 \mathrm{~h}$ bei $-78^{\circ} \mathrm{C}$ gerührt. $7 \Delta^{2} / 7 \Delta^{3}=$ $18 / 82$.

c) Analog b); vor der Silylierung wurde $5 \mathrm{~h}$ bei $-78{ }^{\circ} \mathrm{C}$ gerührt. $7 \Delta^{2} / 7 \Delta^{3}$ $=15 / 85$.

d) Analog b); vor der Silylierung wurde $2 \mathrm{~h}$ bei $-78{ }^{\circ} \mathrm{C}$ und $0.45 \mathrm{~h}$ bei $-45^{\circ} \mathrm{C}$ gerührt. $7 \Delta^{2} / 7 \Delta^{3}=7 / 93$.

e) Analog b); vor der Silylierung wurde $1 \mathrm{~h}$ bei $-20^{\circ} \mathrm{C}$ gerührt. $7 \Delta^{2} / 7 \Delta^{3}$ $<5 / 95$.

\section{Literatur}

1 F. Eiden und K. Th. Wanner, Liebigs Ann. Chem. 1984, 1759.

2 a: R. Knorr und P. Löw, J. Am. Chem. Soc. 102, 3241 (1980). Vgl. hierzu auch b: A. I. Meyers, D. R. Williams, S. White und G. W. Erickson, J. Am. Chem. Soc. 103, 3088 (1981).

3 G. Stork und S. R. Dowd, J. Am. Chem. Soc. 85, 2178 (1963); Vgl. hierzu auch $2 b$.

4 G. Wittig und P. Suchanek, Tetrahedron 1966, 347.

5 H. O. House, L. J. Czuba, M. Gall und H. D. Olmstead, J. Org. Chem. 34, 2324 (1969).

6 Zur Äquilibrierung von Enolaten vgl.: H. O. House und B. M. Trost, J. Org. Chem. 30, 1341, (1965).

7 Bei höheren Äquilibrierungstemp. waren merklich Nebenreaktionen zu beobachten.

8. Vgl. hierzu: F. Johnson, L. D. Duquette, A. Whitehead und L. C. Dorman, Tetrahedron 30, 3241 (1974).

9 J. A. Hirsch und X. L. Wang, Synth. Commun. 12, 333 (1982).

10 M. E. Kuehne und T. Garbacik, J. Org. Chem. 35, 1555 (1970).

11 D. J. Goldsmith, C. M. Dickinson und A. J. Lewis, Heterocycles 25, 291 (1987).

[Ph 360] 